\title{
Studies of Spectral Broadening of the Lower Hybrid Wave Line in the Current-Drive Regimes and Ion Heating at the FT-2 Tokamak
}

\author{
V. V. Dyachenko ${ }^{a}$, , A. B. Altukhov ${ }^{a}$, E. Z. Gusakov ${ }^{a}$, L. A. Esipov ${ }^{a}$, A. N. Konovalov ${ }^{a}$, \\ S. I. Lashkul ${ }^{a}$, A. Yu. Stepanov ${ }^{a}$, and S. V. Shatalin ${ }^{a}$ \\ ${ }^{a}$ Ioffe Physical Technical Institute, St. Petersburg, 194021 Russia \\ *e-mail:v.dyachenko@mail.ioffe.ru
}

Received July 31, 2020; revised October 14, 2020; accepted November 14, 2020

\begin{abstract}
The experiments at the FT-2 tokamak are described that were focused on clearing up the role of the parametric decay instabilities in decreasing the generation efficiency of the non-inductive current excited by the electromagnetic waves in the lower hybrid frequency range. The most discussed instability of such kind is the decay of the pump wave into the daughter high-frequency waves and the low-frequency ion-acoustic quasi-modes. The studies performed have shown that, under conditions of the FT-2 experiment, the ionacoustic instability has no decisive effect on the decrease in the efficiency of the lower hybrid current drive.
\end{abstract}

Key words: tokamak, lower-hybrid current drive, parametric decay instabilities

DOI: $10.1134 / \mathrm{S} 1063780 X 2104005 \mathrm{X}$

\section{INTRODUCTION}

The experiments on the lower hybrid current drive performed at many tokamaks, have shown a much faster decrease in the current drive efficiency with increasing density [1-5] as compared to the theoretical predictions. This phenomenon is associated with the action of the spurious mechanisms at the plasma periphery, which prevent the LH wave penetration into the axial plasma region. The main processes resulting in the peripheral dissipation of the pump wave energy can be the wave scattering by the density fluctuations [6], the collisional absorption [7], and the parametric decay instabilities (PDIs), which are the subject of numerous studies both in theory $[8,9]$, and in experiments [1-5]. The correlation was revealed between the development of the PDIs and the degradation of the current drive efficiency, but there is no generally accepted interpretation of both the role of the PDIs in this degradation (is it concomitant or dominant) and the specific mechanism and scheme of the decay instability responsible for the current drive termination.

At present, two possible schemes are being discussed for the PDI development observed at different tokamaks. One is the parametric decay of the pump wave into the low-frequency (LF) ion-cyclotron quasi-mode and the daughter high-frequency (HF) wave with the lower frequency $[10,11]$. Another scheme consists in the decay into the LF ion-acoustic (IA) quasi-mode and the HF daughter wave [12, 13]. In the first case, a cascade of the radio-frequency (RF) satellites decreasing in frequency is observed in the spectrum of the plasma RF radiation, which are spaced apart by the frequency interval equal to the ion-cyclotron frequency. In accordance with this scheme, when the cascade splitting of the pump wave occurs, a considerable part of the wave power can be spent on the LF ion-cyclotron oscillations and ion acceleration; so, the pump wave loses its energy. According to another scheme, the pump wave energy is spent on the excitation of the IA oscillations. The pump wave is scattered by these oscillations, and the considerable broadening of the pump wave frequency peak occurs. The threshold RF powers required for the developments of both processes can be low, and as a rule, both processes are localized in the peripheral layers of the tokamak plasma.

A series of experiments was carried out at the FT-2 tokamak to study in detail these parametric decay instabilities in hydrogen and deuterium plasmas. In particular, in [14], the authors tried to consider the effect of the PDI involving the ion-cyclotron quasimodes. In this work, we focus on the frequency broadening of the pump wave peak, which many researchers associate with the parametric excitation of the ionacoustic oscillations and with the current drive degradation.

\section{EXPERIMENT DESCRIPTION}

The interaction of the LH waves with plasma was experimentally studied at the small-sized FT-2 toka$\operatorname{mak}\left(R=0.55 \mathrm{~m}, a=0.08 \mathrm{~m}, B_{T} \leq 3 \mathrm{~T}, I_{\mathrm{p}}=19-40 \mathrm{kA}\right.$, $f_{0}=920 \mathrm{MHz}$, and $\left.P_{\mathrm{RF}}<200 \mathrm{~kW}\right)[15,16]$. For this 

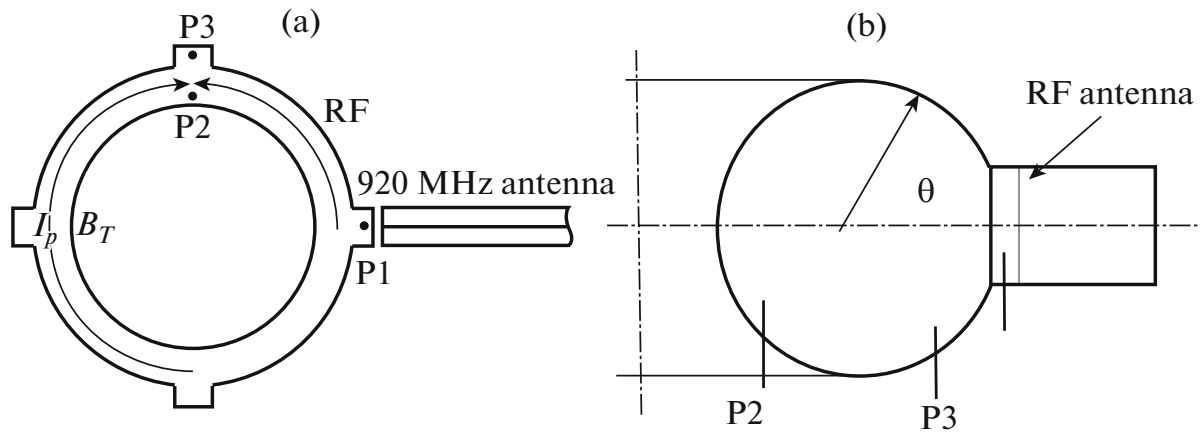

Fig. 1. Schematic of probe arrangement at the FT-2 tokamak. (a) Top view at the tokamak and (b) poloidal sectional view.

purpose, new experimental approaches and the additional diagnostics were used, and the number of the probes used was increased. In the experiments, the magnetron generator was used with the operating frequency $f_{0}=920 \mathrm{MHz}$; its output power $P_{\mathrm{RF}}$ was up to $200 \mathrm{~kW}$ and pulse duration $\tau_{\mathrm{RF}}$ was up to $10 \mathrm{~ms}$. The plasma current and the basic plasma parameters ranged as follows: $I_{\mathrm{p}} \approx 20-30 \mathrm{kA},\left\langle n_{e}\right\rangle=(1-10) \times$ $10^{19} \mathrm{~m}^{-3}, T_{e}(0)=350-400 \mathrm{eV}$, and $T_{i}(0)=80-120 \mathrm{eV}$. The two-waveguide grill was used as the RF antenna. At the $+\pi / 2$ waveguide phasing and a density of $\left\langle n_{e}\right\rangle=3 \times 10^{19} \mathrm{~m}^{-3}$, it generated radiation with the "peak" parameters in the longitudinal slowing-down spectrum of the LH wave introduced into the plasma at $N_{\| 0}=+3.4$ (approximately $41 \%$ of the input power) and $N_{\| 0}=-1.7(19 \%)$. The arrangement of the antenna with respect to the receiving equipment is shown in Figs. 1a and 1b. One of the receiving antennas was the multi-electrode Langmuir probe P3, which was installed in the cross section rotated by $90^{\circ}$ with respect to the RF antenna in the toroidal direction. The probe was installed in the SOL on the low field side (LFS) at a poloidal angle of $\theta=310^{\circ}$ with respect to the equatorial plane of the vacuum chamber. In the same cross section but from the high field side, the single-pin RF probe $\mathrm{P} 2$ was installed $(\theta=$ $\left.220^{\circ}\right)$. The same RF probe $\mathrm{P} 1$ was installed from the LFS in the same cross section as the antenna was installed, under the grill $\left(\theta=320^{\circ}\right)$. The RF signals from the probes were transmitted through the isolation transformers to the spectrum analyzers. The P1 and P2 probes are structurally the same, as well as their circuits transmitting the signals. Most measurements were performed using the Keysight MSOS804A wideband (up to $8 \mathrm{GHz}$ ) digital 4-channel oscilloscope. In the case under consideration, the oscilloscope analyzed the radiation signal in the frequency range from zero to $1.1 \mathrm{GHz}$ over time interval of $200 \mu \mathrm{s}$. When processing and analyzing the data obtained, the presence of spectral components, their time evolution and intensity were determined. Due to the small size of the probes $(l=5 \mathrm{~mm} \ll \lambda$, where $\lambda$ is the radiation wave- length in vacuum), their sensitivity is very low (from -80 to $-40 \mathrm{~dB}$ ) in the frequency range of $0-1 \mathrm{GHz}$, but their frequency response is rather smooth function characterized by a gradual decrease in the probe sensitivities in the low-frequency range. The frequency response of the probes was studied at the broadband diagnostic tester (coaxial section) using the Agilent E5061B network analyzer. In the narrow frequency band $f_{0}=920 \pm 50 \mathrm{MHz}$, the probe sensitivities do not change. Besides the probe measurements, the spectral composition of plasma emission was studied using the reflected signal of the waveguide antenna (high-frequency range), which made it possible to qualitatively compare the observed phenomena. The main information is based on the data obtained from the $\mathrm{P} 1, \mathrm{P} 2$, and $\mathrm{P} 3 \mathrm{RF}$ probes.

\section{EXPERIMENTAL RESULTS}

As noted above, a characteristic feature of the experiments on current drive is the presence of a certain limiting density $\left\langle n_{\text {elim }}\right\rangle$, the so-called density limit $[3-5,13,14]$. The efficient current drive occurs at densities lower than the limiting one, and it quickly terminates when the density limit is exceeded; although the current drive efficiency predicted theoretically should decrease as $1 / n_{e}$ with increasing density [2]. As an example of the "early" current drive degradation, Figs. 2a, 2b, and 2c show the intensity of suprathermal (up to $200 \mathrm{keV}$ ) X-ray radiation from the plasma (the EAST tokamak [5]), the relative drop in the loop voltage and hard X-ray emission intensity (the FT-2 tokamak) as functions of the mean plasma density, respectively. For hydrogen plasma in the FT-2 tokamak, the current drive efficiency abruptly decreases at $\left\langle n_{\text {elim }}\right\rangle \approx 2.5 \times 10^{19} \mathrm{~m}^{-3}$ (for deuterium plasma the density limit is $3 \times 10^{19} \mathrm{~m}^{-3}$ ); for deuterium plasma in the EAST tokamak, at a pump wave frequency of $2.45 \mathrm{GHz}$, the current degradation begins at lower densities $\left\langle n_{\text {elim }}\right\rangle \approx(1-1.5) \times 10^{19} \mathrm{~m}^{-3}$, although the pump wave frequency is much higher. Such a difference in density limits may be caused by the instrumental effect of the signal reduction due to cutting off 


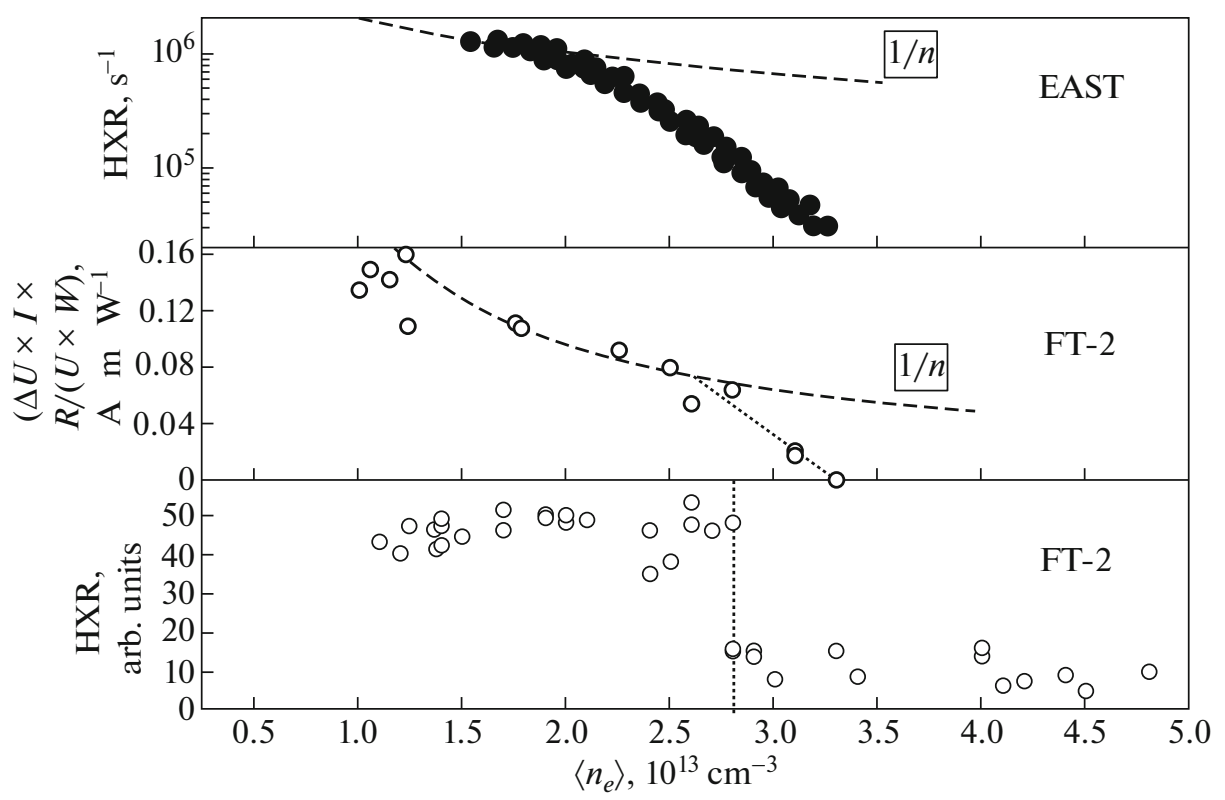

Fig. 2. Comparison between the threshold densities. The following density dependences are shown from top to bottom: the $\mathrm{X}$-ray emission intensity at the EAST tokamak (deuterium, $2.45 \mathrm{GHz}$ ); the current drive efficiency $\Delta U \times I \times R /\left(U \times P_{\mathrm{RF}}\right), \mathrm{A} \mathrm{m} \mathrm{W}^{-1}$ at the FT-2 tokamak (hydrogen, $920 \mathrm{MHz}$ ); and hard X-ray emission intensity at the FT-2 tokamak (hydrogen).

the HXR radiation flow by the filter as the photon energy decreases or it may be associated with the different nature of the mechanisms limiting the current drive efficiency. Therefore, when studying the reasons for the current drive degradation, it is natural to compare the radiation received by the antenna in two operating regimes of the tokamak: the subthreshold regime $\left\langle n_{e}\right\rangle\left\langle\left\langle n_{\text {elim }}\right\rangle\right.$, which is the current drive regime, and the regime with the plasma density higher than the threshold one, $\left.\left\langle n_{e}\right\rangle\right\rangle\left\langle n_{\text {elim }}\right\rangle$, which is the regime when the ion acceleration begins in plasma. Time evolutions of the basic parameters of the FT-2 shot are shown in Fig. 3. The densities of hydrogen plasma before the RF pulse are $\left\langle n_{e}\right\rangle=1 \times 10^{19} \mathrm{~m}^{-3}$ and $3 \times 10^{19} \mathrm{~m}^{-3}$, that is they are lower and higher than the density limit, respectively. For these two densities, the examples of the averaged spectra in the frequency range near the pump wave frequency $f_{0}$, recorded by the $\mathrm{P} 1$ and $\mathrm{P} 2$ probes, are shown in Figs. 4a and 4b. The profile of the pump wave line in the absence of plasma is shown in gray. The averaging was performed as follows: the signal was divided into ten time intervals; for each spectral interval, the area under power spectral density curve was calculated, and the resulting direct images were summed point-to-point and normalized to the number of time intervals. We note here that the signals from different probes (sources) were qualitatively similar. In this case, the location of the probes relative to the radiating antenna is more significant. The $\mathrm{P} 1$ probe is installed in the peripheral plasma in the nearfield region of the antenna, and the plasma has a weak effect on its readings. So, the amplitude of the pump wave peak is almost independent of the plasma density, Fig. 5. Whereas the $\mathrm{P} 2$ and $\mathrm{P} 3$ probes, installed in the cross section rotated by $90^{\circ}$ with respect to the RF antenna (at a distance of $\approx 1 \mathrm{~m}$ ), reflect in more detail the processes in the plasma. In this case, we note that the probes are most sensitive to the waves that are not slowed down or weakly slowed, which propagate in the peripheral plasma. Slow waves from the plasma core can reach the probes due to the secondary processes of scattering, transformation, etc. In addition, the probes have some selectivity with respect to the wave polarization. Therefore, the probe signals should be interpreted rather carefully.

It can be seen that as the wave passes through the plasma, the pump wave peak noticeably broadens in frequency, while the peak amplitude (the P2 probe) decreases with increasing density, Fig. 6. The peak measured by the $\mathrm{P} 1$ probe installed closer to the antenna is considerably less broadened than that measured by the P2 probe installed farther. Quantitative measurements of the $\Delta f_{0}$ peak broadening were carried out at a level of $-10 \mathrm{~dB}$ of the signal maximum. Similar technique of the signal processing was used at other tokamaks (the EAST and the FTU). We note that the narrow peak at the pump wave frequency $\left(\Delta f_{0} \approx\right.$ $0.05 \mathrm{MHz}$ ) is present in all spectra. It can be partly associated with the surface (not slowed) wave, and it can be also a part of the recording circuit noise. When processing the spectra, this peak was digitally cut out.

With increasing plasma density, the $\Delta f_{0}$ broadening first increases, reaching $8 \mathrm{MHz}$ for the $\mathrm{P} 2$ probe; for this probe, no characteristic features in the frequency 


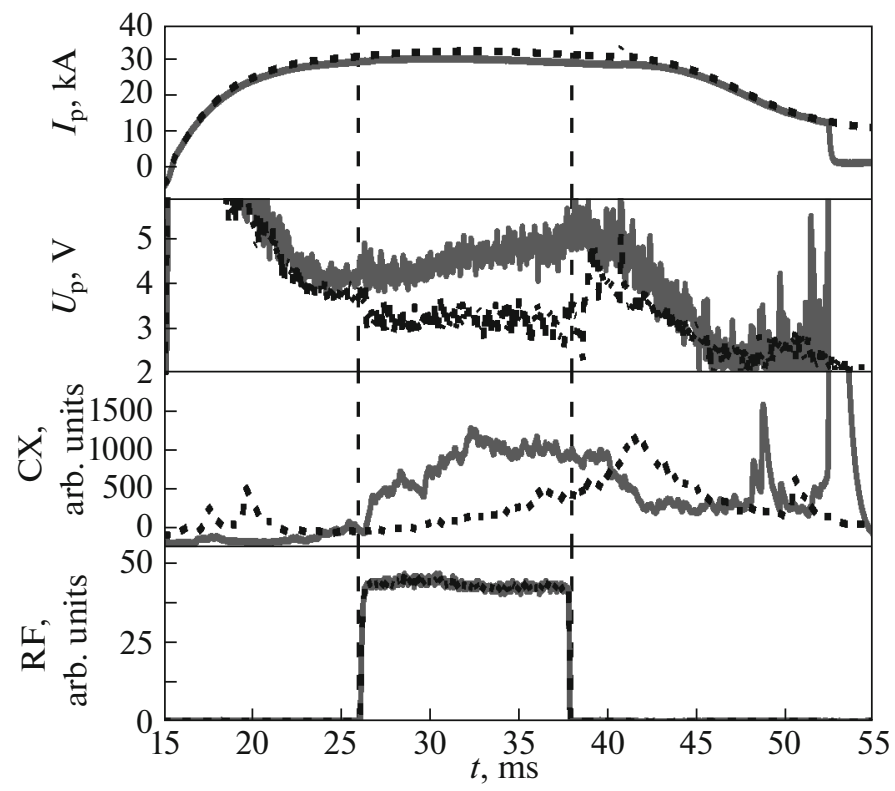

Fig. 3. Time development of hydrogen discharge parameters for two operating regimes of the FT-2 tokamak. The following time dependences are shown from top to bottom: the discharge current, the loop voltage, the flux of charge exchange atoms with $E=980 \mathrm{eV}$, and the RF pulse. Solid and dashed lines correspond to $\left\langle n_{e}\right\rangle=3 \times 10^{19}$ and $1 \times 10^{19} \mathrm{~m}^{-3}$, respectively.

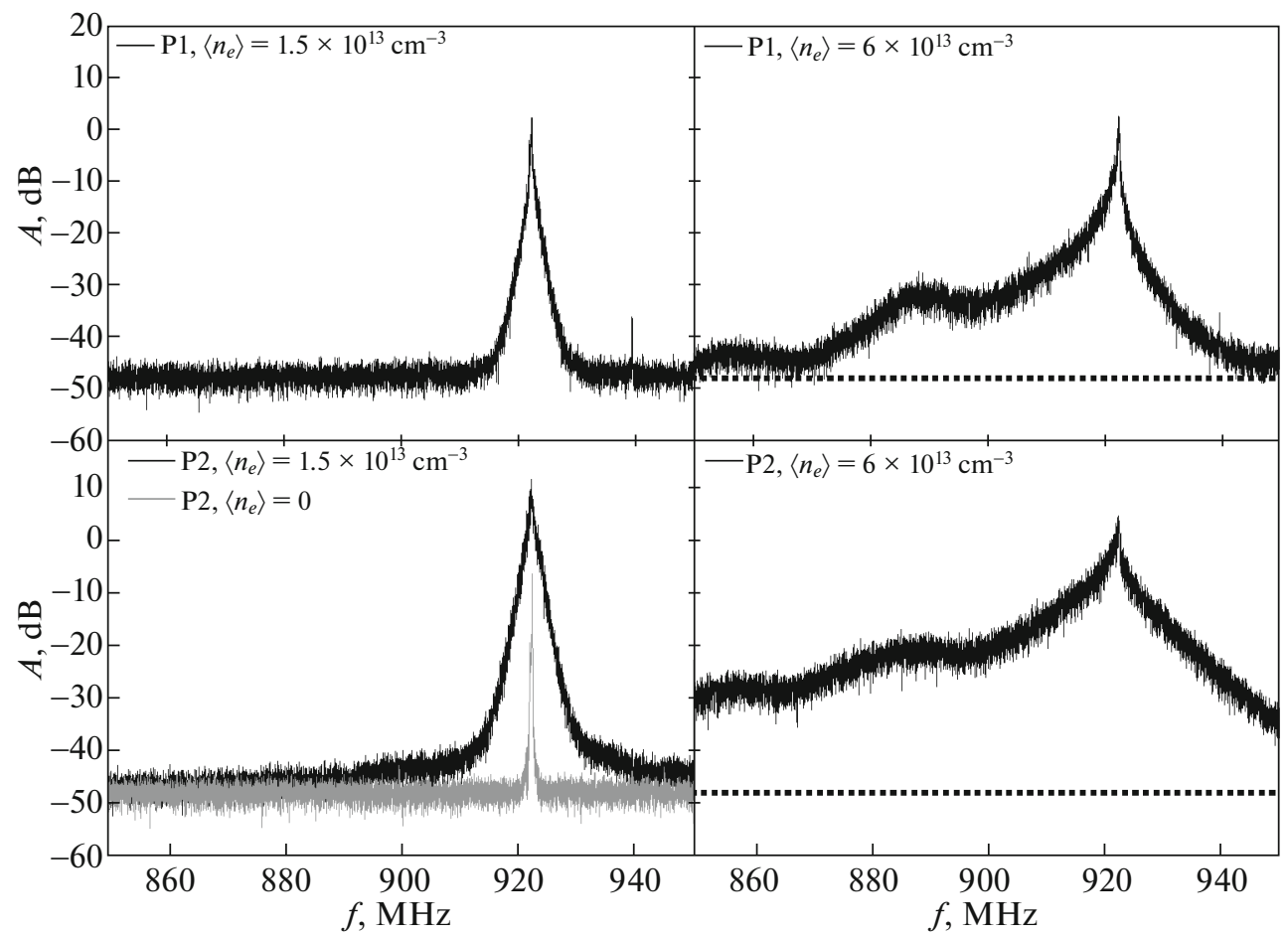

Fig. 4. Frequency spectra of the pump wave peak measured by two probes at two densities: $\left\langle n_{e}\right\rangle=1.5 \times 10^{19}$ and $6 \times 10^{19} \mathrm{~m}^{-3}$. The instrumental contour of the peak in the absence of plasma is shown in gray.

broadening are observed when the density exceeds the density limit, Fig. 6. At densities higher than $(5-6) \times$ $10^{19} \mathrm{~m}^{-3}$, the profile broadening begins to decrease.
We again note here that the broadening considerably depends on the distance between the antenna and the probe: the broadening measured by the $\mathrm{P} 2$ probe 


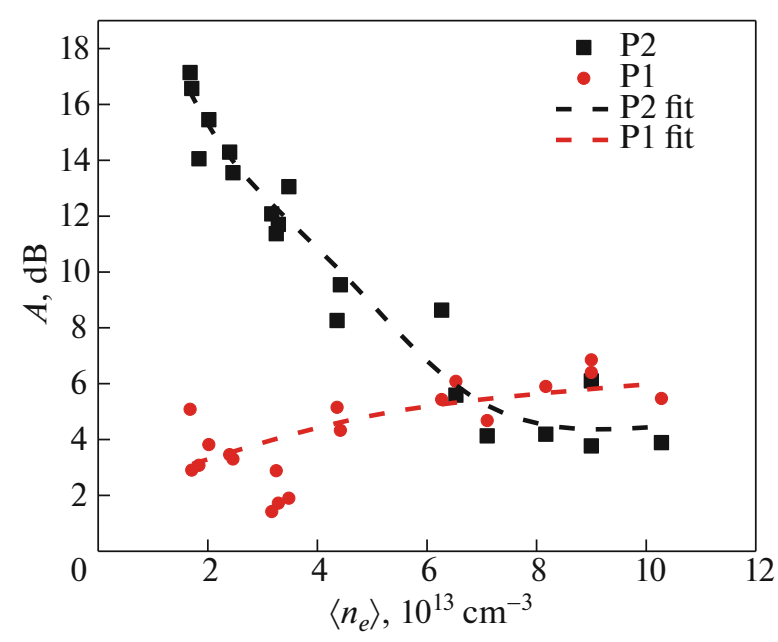

Fig. 5. Amplitudes of the $\mathrm{P} 1$ and $\mathrm{P} 2$ probe signals as functions of hydrogen plasma density. Dashed lines correspond to the approximating curves.

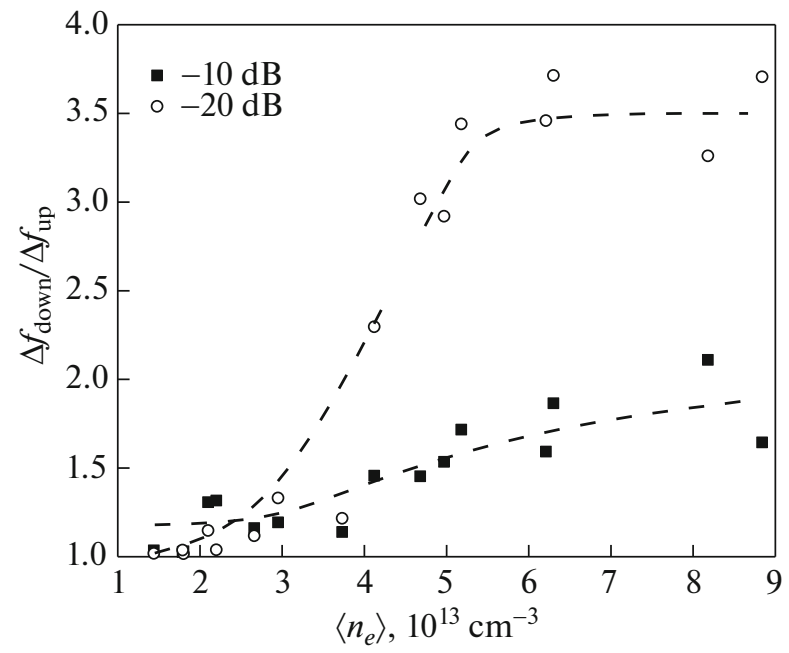

Fig. 7. Asymmetry of the spectra broadening $\Delta f_{\text {down }} / \Delta f_{\text {up }}$ as a function of plasma density. Squares and circles correspond to the measurements performed at levels of $-10 \mathrm{~dB}$ and $-20 \mathrm{~dB}$, respectively.

installed farther is much bigger than that measured by the $\mathrm{P} 1$ probe installed nearer. When working either with hydrogen, or deuterium, no considerable difference in the profile broadening was revealed, although in deuterium plasma, the $\Delta f_{0}$ broadening is always smaller, in accordance with the ion-acoustic dispersion law.

We also note that there is a slight asymmetry between the "red" and "blue" wings of the pump wave peak $\Delta f_{\text {down }} / \Delta f_{\text {up }}$, where $\Delta f_{\text {down }}$ and $\Delta f_{\text {up }}$ are the broadenings in the directions of the "red" and "blue" fre-

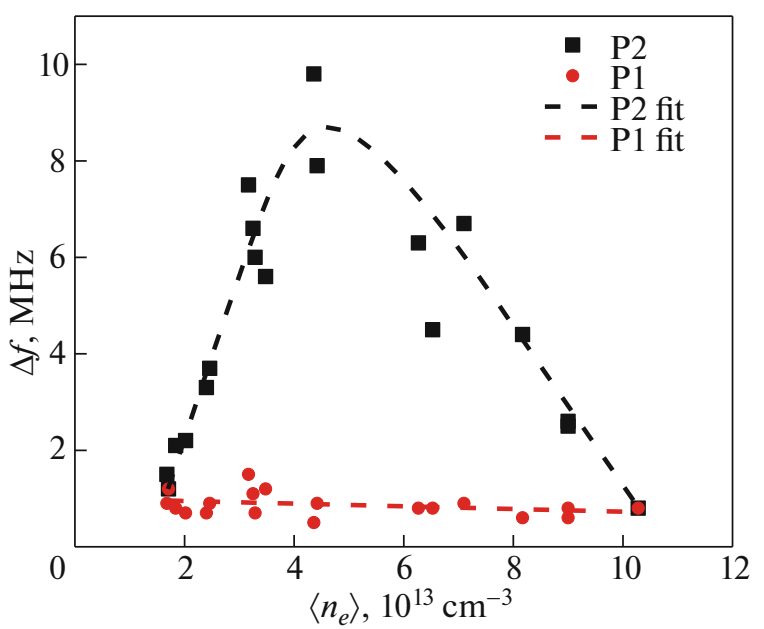

Fig. 6. Peak broadenings $\Delta f_{0}$ as functions of density for two probes: the P1 probe, installed close to the antenna, and the $\mathrm{P} 2$ probe distant from it.

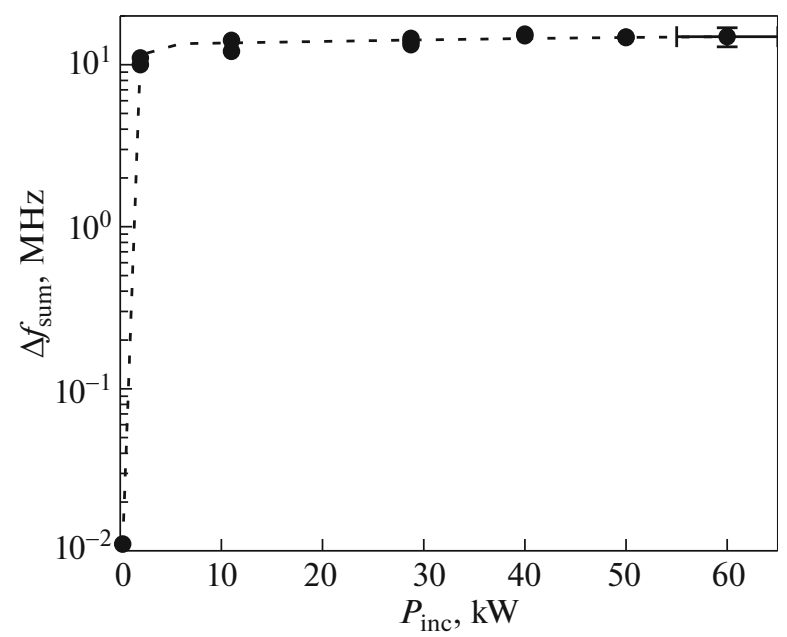

Fig. 8. Peak broadening $\Delta f_{0}$ as a function of the input RF power at a density of $\left\langle n_{e}\right\rangle=4 \times 10^{19} \mathrm{~m}^{-3}$ (the $\mathrm{P} 2$ probe).

quencies from the central frequency $f_{0}$. With increasing density, the "red" wing broadens faster than the "blue" one, Fig. 7. The result observed when measuring the broadening at a level of $-20 \mathrm{~dB}$ of the peak amplitude is completely different. At the densities $\left\langle n_{e}\right\rangle \geq 3 \times 10^{19} \mathrm{~m}^{-3}$, the broadening in the direction of low frequencies (the asymmetry) sharply increases, see Fig. 7.

As the inputted RF power varied in the range of (0-100) $\mathrm{kW}$ (the movable diaphragm ("shutter") was installed in the waveguide line of the antenna [14]) at 
a fixed hydrogen plasma density $\left\langle n_{e}\right\rangle$, the broadening $\Delta f_{0}$ (the $\mathrm{P} 2$ probe) did not considerably change up to powers of $(1-3) \mathrm{kW}$, at which the measurement accuracy becomes no longer sufficient, Fig. 8.

\section{DISCUSSION OF THE RESULTS}

The simplest explanation for the broadening of the pump wave peak would be the wave scattering by the density fluctuations. However, the experiments and numerical simulations using the gyrokinetic code show that the frequencies of the density fluctuations spectra are in the range of $(0.3-0.5) \mathrm{MHz}$, and even at a level of $-30 \mathrm{~dB}$, they do not exceed $1 \mathrm{MHz}$ [17]. Due to this linear mechanism, the peak profile should broaden symmetrically. In the case under consideration, even at low plasma densities, the total peak broadening observed reaches (2-5) MHz. However, at hydrogen plasma densities higher than $3 \times 10^{19} \mathrm{~m}^{-3}$, the $\Delta f_{0}$ broadening reaches $(8-10) \mathrm{MHz}$ (at a level of $-10 \mathrm{~dB}$ ), and the asymmetry of the main peak occurs, which is manifested in the preferential broadening of the "red" wing of the profile (see Fig. 7, squares). At a level of $-20 \mathrm{~dB}$, the asymmetry becomes more pronounced (see Fig. 7, circles). The latter fact can be explained by the parametric excitation of the ioncyclotron quasi-modes, see [14]. In the case under consideration, in hydrogen plasma, this instability starts to develop at plasma densities higher than $(2.5-3) \times 10^{19} \mathrm{~m}^{-3}$. Accordingly, in the vicinity of the pump wave frequency, the lines corresponding to the frequencies of the high-frequency daughter waves $f_{n}=f_{0}-n f_{c i}$ are observed in the signal spectrum. With increasing density, the $\mathrm{Q}$ factor of these peaks decreases; they overlap and merge into one solid pedestal in the "red" frequency range.

A decrease in the amplitude and frequency broadening of the pump wave peak at densities higher than $(4-5) \times 10^{19} \mathrm{~m}^{-3}$ can also be explained in terms of the propagation and absorption of the lower hybrid waves. Numerical simulations show that with increasing density, the minimum longitudinal refractive index required for the propagation of the lower hybrid waves $N_{\text {cr }}$ increases, and the spatial spectrum of waves excited by the grill changes. The fraction of the strongly slowed waves increases, and accordingly, the scattering of waves by plasma oscillations also increases. The ray propagation of the wave acquires a diffusion character, and the resonance cone gets rapidly "smeared" over the plasma column cross section. The characteristic attenuation length of the coherent wave component (due to such scattering) can be written in the following simplified form (see formula (87) in [18]): $l_{s} \sim n_{e}^{-1 / 2} N_{\| 0}^{-1}\left(\delta n_{e}\right)^{-2}$, where $N_{\| 0}$ is the initial slowing factor of the pump wave, and $\delta n_{e}=\Delta n_{e} / n_{e}$ is the level of density fluctuations. According to the gyrokinetic calculations, confirmed experimentally in [19], in the peripheral plasma of the FT-2 tokamak, the level of density fluctuations is $\Delta n_{e} / n_{e} \approx(1-2) \times$ $10^{-1}$, and in the core plasma, it is $\Delta n_{e} / n_{e} \approx 2 \times 10^{-2}$. Then the attenuation length is $l_{s} \approx 1 \mathrm{~m}$ already at $\left\langle n_{e}\right\rangle \approx$ $2.5 \times 10^{19} \mathrm{~m}^{-3}$ and $N_{\| 0} \approx 3$. Only a small fraction of the wave energy reaches the distant probes; therefore, they detect only a part of the pump wave propagating in the cold peripheral plasma; accordingly, the observed frequency broadening is small (of the order of 1$2 \mathrm{MHz}$ ). With increasing density, this effect becomes dominant. However, serious numerical simulations are required to confirm this explanation. For the same reason, in the regions far from the antenna, the probability of the PDI development decreases due to a decrease in the wave electric field strength. It will also decrease if we take into account the effect of the collisional absorption, which strongly suppresses the PDI growth rates [20].

Many authors associate the observed considerable broadening of the pump wave peak, which exceeds the density fluctuation frequency, with the PDI excitation. In this process, the ion-acoustic quasi-mode acts as a low-frequency branch $[5,12,13]$. In the short-wave region of the dispersion dependence $\left(k r_{\mathrm{D}} \gg 1, k\right.$ is the wave number, and $r_{\mathrm{D}}$ is the Debye radius), the ion-acoustic wave is transformed into the non-propagating plasma oscillations with the possible frequencies up to the ion plasma frequency $\omega_{p i}$. If we restrict ourselves to the case $T_{e} \sim T_{i}$, in the low-frequency range, the dispersion equation for the IA quasi-mode can be written as $\omega_{i s} \approx \omega_{0} N_{\|} V_{\text {th }, i} / c$ [12], where $V_{\text {th }, i}$ is the ion thermal velocity, and $N_{\|}$is the longitudinal component of the refractive index of the daughter IA wave.

According to numerical modeling [12], the growth rate of this instability is maximal in the range $N_{\|} \approx 4-$ 8 , which for the peripheral temperature $T_{i} \sim 10-100 \mathrm{eV}$ available in the experiment, corresponds to the IA frequency of the order of (0.5-3.0) $\mathrm{MHz}$. At the same time, the maximum broadening measured in experiment reaches $8 \mathrm{MHz}$, which is slightly higher than the expected broadening. This may indicate the generation of the IA waves with large longitudinal slowing factors (of the order of 10), especially as such $N_{\|}$components are present in the spatial spectrum of the emitted waves.

As for the threshold dependence on the RF power, there are different theoretical predictions that depend on the assumptions concerning the structure of the pump wave field and the mechanisms for the energy transport from the region of the wave-plasma interaction. According to different estimates, for a given type of decay processes, the threshold power can vary from $1 \mathrm{~kW}$ to $1 \mathrm{MW}$, depending on the plasma parameters and the slowing factor of the daughter wave (see, e.g., [12]). The experimental dependence obtained in this study (Fig. 8) indicates that the RF power threshold is 
either absent at all, or it does not exceed 1-2 kW, whereas at the EAST tokamak [5], the degradation of the current drive efficiency begins at an input power of $550 \mathrm{~kW}$ at a frequency of $2.45 \mathrm{GHz}$ (experimental threshold). Taking into account the huge difference in the antenna sizes used at the EAST and FT-2 tokamaks, it is more correct to discuss the results in terms of the magnitude of the high-frequency field strength in the antenna aperture, $\tilde{E}$. For the EAST, we estimated the $\tilde{E}$ field to be approximately $1.5 \mathrm{kV} \mathrm{cm}^{-1}$ at a power of $550 \mathrm{~kW}(2.45 \mathrm{GHz})$, and for the FT-2, it is $1-1.5 \mathrm{kV} \mathrm{cm}^{-1}$ at an input power of $100 \mathrm{~kW}$; so, the conditions for the PDI development are approximately the same. Nevertheless, at the FT-2, the current drive degradation begins at a density of $\left\langle n_{e}\right\rangle=$ $2.5 \times 10^{19} \mathrm{~m}^{-3}$, and at the EAST tokamak, it begins at much lower densities of $\left\langle n_{e}\right\rangle=1.0 \times 10^{19} \mathrm{~m}^{-3}$. Apparently, the X-ray diagnostics at the EAST tokamak is more sensitive to changes in the distribution function of suprathermal electrons accelerated by the $\mathrm{LH}$ wave than the loop voltage diagnostics at the FT-2.

\section{CONCLUSIONS}

Thus, the noticeable frequency broadening of the pump wave peak is observed when the LH power is injected into the tokamak. This broadening considerably exceeds the frequencies of the plasma density fluctuations, but qualitatively coincides with the frequencies characteristic of the ion-acoustic quasimodes, the development of which can be induced by the LH wave. The peak broadening depends on the plasma density, while there is almost no threshold with respect to the input power, which is characteristic of the PDI development. When the current drive regime is changed to the ohmic confinement regime with ion acceleration, no specific features are observed in the broadening magnitude or nature. This gives reason to believe that, in contrast to the experiment at the EAST tokamak, under the conditions of the FT-2 tokamak, the development of the ion-acoustic parametric instability (quasi-mode) does not have a fatal effect on the current drive efficiency. In the case of the experiment at the FT-2 tokamak, the large broadenings of the pump wave spectral line observed (up to $10 \mathrm{MHz}$ ) can be partly explained by the increased sensitivity of the measuring equipment (probes are installed in plasma, as opposed to the EAST facility) and the multiple scattering of the LH waves. We explain the observed "early" current drive termination at densities higher than $2.5 \times 10^{19} \mathrm{~m}^{-3}$ by the development of another PDI, namely, by the development of the ion cyclotron quasi-mode described in [14]. At high densities, it can also lead to the formation of a low-frequency wing in the pump wave spectrum.

\section{ACKNOWLEDGMENTS}

The authors are grateful to A.D. Gurchenko for the information provided on the level of density fluctuations at the FT-2 tokamak and their frequency spectra.

\section{FUNDING}

The work was supported by the Ioffe Institute under the State Assignment. The information on the frequencies and amplitudes of the density fluctuations in plasma of the FT2 tokamak, which was used in Section 4, was obtained in experiments supported by the Russian Science Foundation (project no. 17-12-01110).

\section{CONFLICT OF INTEREST}

The authors declare that they have no conflict of interests.

\section{OPEN ACCESS}

This article is licensed under a Creative Commons Attribution 4.0 International License, which permits use, sharing, adaptation, distribution and reproduction in any medium or format, as long as you give appropriate credit to the original author(s) and the source, provide a link to the Creative Commons licence, and indicate if changes were made. Theimages or other third party material in this article are included in thearticle's Creative Commons licence, unless indicated otherwise in a credit line to the material. If material is not included in the article's Creative Commons licence and your intended use is not permitted by statutory regulation or exceeds the permitted use, you will need to obtain permission directly from the copyright holder. To view a copy of this licence, visit http://creativecommons.org/licenses/by/4.0/.

\section{REFERENCES}

1. V. N. Budnikov, V. V. Dyachenko, M. A. Irzak, E. R. Its, S. I. Lashkul, K. A. Podushnikova, A. Yu. Stepanov, O. N. Shcherbinin, and M. J. Vildjunas, in Proceedings of the 22nd EPS Conference on Controlled Fusion and Plasma Physics, Bournemouth, 1995, ECA 19C (4), IV-385 (1995).

2. V. Pericoli-Ridolfini, L. Giannone, and R. Bartiromo, Nucl. Fusion 34, 469 (1994).

3. G. M. Wallace, R. R. Parker, P. T. Bonoli, A. E. Hubbard, J. W. Hughes, B. L. LaBombard, O. Meneghini, A. E. Schmidt, S. Shiraiwa, D. G. Whyte, J. C. Wright, S. J. Wukitch, R. W. Harvey, A. P. Smirnov, and J. R. Wilson, Phys. Plasmas 17, 082508 (2010).

4. M. Goniche, L. Amicucci, Y. Baranov, V. Basiuk, G. Calabro, A. Cardinali, C. Castaldo, R. Cesario, J. Decker, D. Dodt, A. Ekedahl, L. Figini, J. Garcia, G. Giruzzi, J. Hillairet, et al., Plasma Phys. Control. Fusion 52, 124031 (2010).

5. M. H. Li, B. J. Ding, F. K. Liu, J. F. Shan, M. Wang, H. D. Xu, R. Cesario, F. Napoli, C. Castaldo, A. Cardinali, L. Liu, L. M. Zhao, H. C. Hu, X. J. Zhang, 
Y. C. Li, et al., Plasma Phys. Control. Fusion 61, 065005 (2019).

6. P. Andrews and F. Perkins, Phys. Fluids 26, 2546 (1983).

7. B. V. Galaktionov, V. V. D’yachenko, M. M. Larionov, and O. N. Shcherbinin, Sov. Phys.-Tech. Phys. 19, 459 (1974).

8. M. Porkolab, Nucl. Fusion 18, 367 (1978).

9. R. Cesario, L. Amicucci, A. Cardinali, C. Castaldo, M. Marinucci, F. Napoli, F. Paoletti, D. De Arcangelis, M. Ferrari, A. Galli, G. Gallo, E. Pullara, G. Schettini, and A. A. Tuccillo, Nucl. Fusion 54, 043002 (2014).

10. V. N. Budnikov, V. V. Dyachenko, L. A. Esipov, S. I. Lashkul, V. A. Aleksandrov, I. E. Saharov, and S. V. Shatalin, in Proceedings of the 19th EPS Conference on Controlled Fusion and Plasma Physics, Innsbruck, 1992, ECA 16C, 997 (1992).

11. Y. Takase, M. Porkolab, J. Schuss, R. Watterson, C. Fiore, R. E. Slusher, and C. M. Surko, Phys. Fluids 28, 983 (1985).

12. R. Cesario and A. Cardinali, Nucl. Fusion 29, 1709 (1989).

13. Y. Takase and M. Porkolab, Phys. Fluids 26, 2992 (1983).

14. V. V. D’yachenko, A. N. Konovalov, A. Yu. Stepanov, A. B. Altukhov, E. Z. Gusakov, L. A. Esipov,
S. I. Lashkul, and S. V. Shatalin, Plasma Phys. Rep. 45, 1134 (2019).

15. S. I. Lashkul, A. B. Altukhov, A. D. Gurchenko, V. V. D'yachenko, L. A. Esipov, M. Yu. Kantor, D. V. Kuprienko, M. A. Irzak, A. N. Savel'ev, A. V. Sidorov, A. Yu. Stepanov, and S. V. Shatalin, Plasma Phys. Rep. 36, 751 (2010).

16. S. I. Lashkul, A. B. Altukhov, A. D. Gurchenko, E. Z. Gusakov, V. V. D'yachenko, L. A. Esipov, M. A. Irzak, M. Yu. Kantor, D. V. Kuprienko, A. N. Savel'ev, A. Yu. Stepanov, and S. V. Shatalin, Plasma Phys. Rep. 41, 990 (2015).

17. P. Niskala, A. D. Gurchenko, E. Z. Gusakov, A. B. Altukhov, L. A. Esipov, M. Yu. Kantor, T. P. Kiviniemi, D. V. Kouprienko, T. Korpilo, S. I. Lashkul, S. Leerink, A. A. Perevalov, and R. Rochford, Plasma Phys. Control. Fusion 59, 044010 (2017).

18. R. L. Berger, Liu Chen, P. K. Kaw, and F. W. Perkins, Phys. Fluids 20, 1864 (1977).

19. A. B. Altukhov, A. D. Gurchenko, E. Z. Gusakov, M. A. Irzak, P. Niskala, L. A. Esipov, T. P. Kiviniemi, O. L. Krutkin, and S. Leerink, Phys. Plasmas 25, 112503 (2018).

20. C. Castaldo, A. Di Siena, R. Fedele, F. Napoli, L. Amicucci, R. Cesario, and G. Schettini, Nucl. Fusion 56, 016003 (2016).

Translated by I. Grishina 